\title{
ON SUPPORTLESS CONVEX SETS IN INCOMPLETE NORMED SPACES
}

\author{
V. P. FONF \\ (Communicated by Dale Alspach)
}

\begin{abstract}
It is proved that every incomplete separable normed space $M$ contains a closed bounded convex set $W$ such that the closed linear span of $W$ coincides with $M$ and $W$ contains no weakly supported points. This theorem answers a question of Klee and a question of Borwein and Tingley.
\end{abstract}

Let $A$ be a subset of normed space $X$. A point $x \in A$ is called a support point of $A$ if there exists a nonzero continuous linear functional $f \in X^{*}$ such that $f(x)=\sup f(A)$. We shall call a point $x \in A$ a weakly supported point if there exists a linear functional $f$ such that the restriction $f / A$ is nonzero and continuous and $f(x)=\sup f(A)$. The last definition was inspired by the paper of Klee [1]. The set of all support points of the set $A$ (respectively weakly supported points) we denote by $\operatorname{supp} A$ (respectively wsupp $A$ ). In $1958 \mathrm{Klee}$ [2] gave a striking example of a convex bounded closed subset $A$ in a certain incomplete normed space $X$ (actually $X$ is a dense subspace of $\ell_{2}$ ) possessing the property $\operatorname{supp} A=\varnothing$ (a supportless set). Subsequently, Bishop and Phelps [3] proved that for every closed bounded convex subset $A$ (a CBC subset) of the Banach space $X$ the set of all support functionals $\sum(A)$ of the set $A$ is dense in dual space $X^{*}$. Later in [4] they proved that for every CBC subset $A$ of a Banach space the set $\operatorname{supp} A$ is dense in the boundary of the set $A$. Hence supportless CBC subsets may occur only in incomplete normed spaces. In 1985 Borwein and Tingley [5], developing ideas of Klee, constructed in every separable Banach space a dense subspace possessing a supportless CBC subset (additionally, their set is absorbing; see the paper [6] of the author where he got a characterization of incomplete normed spaces possessing supportless absorbing CBC subsets). The main conjecture (formulated in paper [5] which inspired our investigation) is that every incomplete normed space contains a supportless CBC subset. We shall prove this conjecture (actually in a stronger form, which permits us to answer a question of Klee concerning weakly supported points [1]).

Received by the editors July 21, 1992.

1991 Mathematics Subject Classification. Primary 46B20. 
First, we need an auxiliary lemma. But let us remember that a system $\left\{x_{i}\right\}$ in a Banach space $X$ is called an $M$-basis of the space $X$ if $\left\{x_{i}\right\}$ is the minimal complete system with total biorthogonal system [7].

Lemma. Let $M$ be a dense proper subspace of the separable Banach space $X$. Then there exist an $M$-basis $\left\{x_{i}\right\}_{1}^{\infty} \subset M$ of the space $X$ such that $\left\|x_{i}\right\| \leq$ $2^{-i}, i=1,2, \ldots$, and a sequence $\left\{\xi_{i}\right\}_{1}^{\infty}, \sum\left|\xi_{i}\right|=1$, such that $\sum \xi_{i} x_{i} \notin M$.

Proof. Subsequently using an existense of an $M$-basis in every separable Banach space [8], the Krein-Milman-Rutman stability theorem [9], and density of $M$ in the space $X$ we can choose an $M$-basis $\left\{x_{i}\right\}$ of the space $X$ in the subspace $M$. In addition we suppose that $\left\|x_{i}\right\| \leq 2^{-i-1}$ for $i=1,2, \ldots$. If there exists a sequence $\left\{\xi_{i}\right\}$ with $\sum\left|\xi_{i}\right|=1$ such that $\sum \xi_{i} x_{i} \notin M$ the proof is ended. Otherwise (i.e., for every $\left\{\eta_{i}\right\}$ with $\sum\left|\eta_{i}\right|=1$ we have $\sum \eta_{i} x_{i} \in$ $M$ ) let $\left\{\varepsilon_{i}\right\}$ be the decreasing sequence of stability of the $M$-basis $\left\{x_{i}\right\}$ [9]. Without loss of generality we can assume that $0<\varepsilon_{i}<2^{-i-1}, i=1,2, \ldots$. Let $y \in X \backslash M,\|y\| \leq \varepsilon_{1}^{2} / 2$. Hence by density of $M$ in $X$ there exists a sequence $\left\{z_{i}\right\}_{0}^{\infty}, z_{0}=0$, such that $\left\|y-z_{i}\right\| \leq \varepsilon_{i+1}^{2} / 2, i=1,2, \ldots$. We have $\left\|z_{i}-z_{i-1}\right\| \leq \varepsilon_{i}^{2}, i=1,2, \ldots$. Let $x_{i}^{\prime}=x_{i}+1 / \varepsilon_{i}\left(z_{i}-z_{i-1}\right), i=1,2, \ldots$. Since $\left\|1 / \varepsilon_{i}\left(z_{i}-z_{i-1}\right)\right\| \leq \varepsilon_{i}$, it follows that $\left\{x_{i}^{\prime}\right\}$ is an $M$-basis of the space $X$. Thus we have

$$
\begin{aligned}
y^{\prime} & =\sum \frac{\varepsilon_{i}}{\sum \varepsilon_{j}} x_{i}^{\prime}=\frac{1}{\sum \varepsilon_{j}} \sum \varepsilon_{i} x_{i}+\frac{1}{\sum \varepsilon_{j}} \sum\left(z_{i}-z_{i-1}\right) \\
& =\frac{1}{\sum \varepsilon_{j}} \sum \varepsilon_{i} x_{i}+\frac{1}{\sum \varepsilon_{j}} y .
\end{aligned}
$$

Since $\sum \varepsilon_{i} x_{i} / \sum \varepsilon_{j} \in M$ (by assumption) and $y \notin M$, it follows that $y^{\prime} \notin M$. Setting $\xi_{i}=\varepsilon_{i} / \sum \varepsilon_{j}, i=1,2, \ldots$, we complete the proof.

The following theorem gives an answer to one of the main open questions in the paper [5] by Borwein and Tingley.

Theorem. Every incomplete separable normed space $M$ contains a closed bounded convex subset $W$ such that the closed linear span of $W$ coincides with $M$ and $W$ contains no weakly supported points, i.e.,

$$
\text { wsupp } W=\varnothing \text {. }
$$

Proof. Let $X$ be the completion of $M$ and $\left\{x_{i}\right\} \subset M$ be an $M$-basis of the space $X$ possessing the properties that (see the lemma) $\left\|x_{i}\right\| \leq 2^{-i}$ for $i=$ $1,2, \ldots$ and there exists a sequence $\left\{\xi_{i}\right\}$ such that $\sum\left|\xi_{i}\right|=1$ and $y=$ $\sum \xi_{i} x_{i} \notin M$. Let us define an operator $T: \ell_{1} \rightarrow X$ by $T e_{i}=x_{i}, i=1,2, \ldots$, where $\left\{e_{i}\right\}$ is the canonical basis of the space $\ell_{1}$. So $T$ is an injective compact operator, and the image $V=T U\left(\ell_{1}\right)$ of the unit ball $U\left(\ell_{1}\right)$ of the space $\ell_{1}$ is closed. Let $W=M \cap\left(-\frac{1}{2} y+V\right)$, and introduce the following notation:

$$
L=T^{-1}(M), \quad z=T^{-1} y=\sum \xi_{i} e_{i}, \quad A=T^{-1}(W)=L \cap\left(-\frac{1}{2} z+U\left(\ell_{1}\right)\right) .
$$

By density of the set $\operatorname{lin}\left\{e_{i}\right\}$ in the space $\ell_{1}$ we have

$$
\operatorname{cl}\left(A \cap \operatorname{lin}\left\{e_{i}\right\}\right)=-\frac{1}{2} z+U\left(\ell_{1}\right) .
$$


Therefore,

$$
\operatorname{cl} \operatorname{lin} A=l_{1} .
$$

Hence

$$
\operatorname{cllin} W=\operatorname{cl} \operatorname{lin} T A=X
$$

and

$$
\operatorname{cl}\left(W \cap \operatorname{lin}\left\{x_{i}\right\}\right) \supset W .
$$

Now suppose that a linear functional $f$ on $M$ exists such that the restriction $f / W$ is continuous and nonzero and $f$ attains its supremum on the set $W$ at some point $x_{0} \in W: f\left(x_{0}\right)=\sup f(W)$. Without loss of generality we can assume that the functional $f$ is defined on the whole space $X$. Denote by $\hat{f}$ the linear functional on the space $\ell_{1}$ defined by $\hat{f}(u)=f(T u), u \in \ell_{1}$. It is easily verified that $\hat{f} / A$ is $w^{*}$-continuous in the duality $c_{0}^{*}=\ell_{1}$ (remember that $f / W$ is continuous, and note that the operator $T / A:\left(A, w^{*}\right) \rightarrow W$ is continuous as the restriction of an adjoint operator). Since $\frac{1}{2} e_{i}=-\frac{1}{2} z+\left(\frac{1}{2} z+\frac{1}{2} e_{i}\right) \in A$ and $w^{*}-\lim e_{i}=0, \lim \hat{f}\left(e_{i}\right)=0$. As $f / W$ is a nonzero continuous functional, (4) gives the existence of an integer $j$ such that $f\left(x_{j}\right) \neq 0$, so $\hat{f}\left(e_{j}\right) \neq 0$. Now let $h$ be the element of the space $c_{0}$ such that $h\left(e_{i}\right)=\hat{f}\left(e_{i}\right), \quad i=1,2, \ldots$. Obviously, $h \neq 0$, and

$$
h / \operatorname{lin}\left\{e_{i}\right\}=\hat{f} / \operatorname{lin}\left\{e_{i}\right\}
$$

Moreover, $h / A=\hat{f} / A$. Indeed let $v \in A, v=-\frac{1}{2} z+\sum \gamma_{i} e_{i}, \sum\left|\gamma_{i}\right| \leq 1$. Since $v \in L, \operatorname{lin}\left\{e_{i}\right\} \subset L$, and $-\frac{1}{2} z \notin L$, it follows that the sum $\sum \gamma_{i} e_{i}$ contains infinitely many nonzero members. Therefore, for each positive integer $m$ there is a positive integer $n_{m}$ such that $\frac{1}{2} \sum_{n_{m}+1}^{\infty}\left|\xi_{i}\right|<\sum_{m+1}^{\infty}\left|\gamma_{i}\right|$. Let $z_{m}=$ $-\frac{1}{2} \sum_{1}^{\infty} \xi_{i} e_{i}+\sum_{1}^{m} \gamma_{i} e_{i}+\frac{1}{2} \sum_{n_{m}+1}^{\infty} \xi_{i} e_{i}$. Then $z_{m} \in A$, and $z_{m}=-\frac{1}{2} \sum_{1}^{n_{m}} \xi_{i} e_{i}+$ $\sum_{1}^{m} \gamma_{i} e_{i} \in \operatorname{lin}\left\{e_{i}\right\}$. Thus, by (5), $h\left(z_{m}\right)=\hat{f}\left(z_{m}\right)$, but $\lim z_{m}=v$, so $\hat{f}(v)=$ $\lim \hat{f}\left(z_{m}\right)=\lim h\left(z_{m}\right)=h(v)$. From $\hat{f} / A=h / A$ we easily get

$$
\sup \hat{f}(A)=\sup h(A)=\sup h(\operatorname{cl} A) .
$$

Let $g=T^{-1} x_{0}=-\frac{1}{2} z+\sum a_{i} e_{i}$ where $\sum\left|a_{i}\right| \leq 1$. Hence $g \in A$, and by (6) and (1) we have

$$
\begin{aligned}
h(g) & =\hat{f}(g)=f\left(x_{0}\right)=\sup f(W)=\sup \hat{f}(A) \\
& =\sup h(A)=\sup h(\operatorname{cl} A)=\sup h\left(-\frac{1}{2} z+U\left(\ell_{1}\right)\right) .
\end{aligned}
$$

Thus $h\left(\sum a_{i} e_{i}\right)=\sup h\left(U\left(\ell_{1}\right)\right)$. Since $h \in c_{0}$, we see that the sum $\sum a_{i} e_{i}$ contains only a finite number (say, $m$ ) of nonzero members. So $x_{0}=-\frac{1}{2} y+$ $\sum_{1}^{m} a_{i} x_{i}$, but $x_{0} \in W \subset M$ and $\sum_{1}^{m} a_{i} x_{i} \in M$ while $y \notin M$. This contradiction completes the proof.

The following corollary is a simple combination of the Theorem with the previously mentioned famous results of Bishop and Phelps. 
Corollary 1. Let $X$ be a separable normed space. The following assertions are equivalent:

(1) Every $C B C$ subset $W \subset X$ has a support point.

(2) Every $C B C$ subset $W \subset X$ has its support points dense in the boundary of the set $W$.

(3) Every $C B C$ subset $W \subset X$ has a support functional $f \in X^{*}$.

(4) For every $C B C$ subset $W \subset X$ the set of all support functionals $\sum(W)$ of the set $W$ is dense in the dual space $X^{*}$.

(5) The space $X$ is complete (i.e., $X$ is a Banach space).

The next corollary gives an answer to a question of Klee [1].

Corollary 2. Every incomplete separable normed space $M$ contains a dense subspace $M_{1}$ possessing a closed (even in $M$ ) bounded convex subset $W$ such that each nonzero linear functional $f$ on $M_{1}$ with continuous restriction $f / W$ does not attain its supremum on the set $W$.

Proof. Let $W$ be a subset constructed as in the theorem, and let $M_{1}=\operatorname{lin} W$. Then $W$ and $M_{1}$ are as required.

We conclude the paper with the following open question.

Question. Does every incomplete (separable) normed space $M$ contain a closed bounded convex subset $W$ such that each nonzero linear functional $f$ on $M$ with continuous restriction $f / W$ does not attain its supremum on the set $W$ ?

\section{ACKNOWLEDGMENT}

The author wishes to thank the referee for a series of suggestions which essentially improved the paper.

\section{REFERENCES}

1. V. Klee, On a question of Bishop and Phelps, Amer. J. Math. 85 (1963), 95-98.

2. __ Extremal structure of convex sets. II, Math. Z. 69 (1958), 90-104.

3. E. Bishop and R. R. Phelps, A proof that every Banach space is subreflexive, Bull. Amer. Math. Soc. 67 (1961), 97-98.

4. The support functionals of a convex set, Convexity, Proc. Sympos. Pure Math., vol. 7, Amer. Math. Soc., Providence, RI, 1963, pp. 27-35.

5. J. M. Borwein and D. W. Tingley, On supportless convex sets, Proc. Amer. Math. Soc. 94 (1985), 471-476.

6. V. P. Fonf, On absorbing supportless convex subsets in normed spaces, Studia Math. 104 (1993), 279-284.

7. I. Singer, Bases in Banach spaces, Vol. 2, Springer-Verlag, Berlin, 1981.

8. A. I. Markushevich, On basis (in the broad sense of the word) for linear spaces, Dokl. Akad. Nauk SSSR 41 (1943), no. 6, 241-244.

9. M. G. Krein, D. P. Milman, and M. A. Rutman, On one property of basis in a Banach space, Notes of Math. Soc., Kharkov 16 (1940), 106-110.

Department of Mathematics, Kharkov Institute of Railway Engineers, SQ. FeujerBACH 7, 310050 KHARKOV, UKRAINE

Current address: Department of Mathematics and Computer Science, Ben-Gurion University of the Negev, Beer-Sheva, Israel 